Chinese Journal of Organic Chemistry

\title{
$L$-鸟嘌呤异核苷的全合成研究
}

\author{
唐杰董祥有欧阳文良朱云龙海新* 肖强* \\ (江西科技师范大学有机功能分子研究所 江西省有机功能分子重点实验室 南昌 330013)
}

\begin{abstract}
摘要 发展了一条改进的 $L$-鸟嘌呤异核苷全合成路线. 以 $L$-核糖为起始原料，合成了 3,5-O-二芐基-1-脱氧- $L-$-核糖，再 与碱基 $N^{2}, N^{2}$-二叔丁氧羰基-6-氯鸟嘌呤发生关键的 Mitsunobu 反应来合成异核苷 6. 经过 9 步反应, 以 $37.3 \%$ 的总收率 合成了 $L$-鸟嘌呤异核苷, 其中 Mitsunobu 反应构建异核苷键具有立体专一性、高产率、条件温和、区域选择性高等优 点. 该方法可以作为鸟嘌呤异核苷的通用合成路线.
\end{abstract}

关键词 核苷; 全合成; 糖基化; Mitsunobu 反应; 异核苷

\section{Studies on the Total Synthesis of iso-L-Guanosine}

\author{
Tang, Jie \\ Dong, Xiangyou \\ Ouyang, Wenliang \\ Zhu, Yunlong \\ Ding, Haixin* \\ Xiao, Qiang* \\ (Jiangxi Key Laboratory of Organic Chemistry, Institute of Organic chemistry, Jiangxi Science \& Technology \\ Normal University, Nanchang 330013)
}

\begin{abstract}
An improved route for the total synthesis of iso- $L$-guanosine was developed. Using $L$-ribose as the starting material, 3,5-O-dibenzyl-1-deoxy- $L$-ribose was firstly synthesized. Then, Mitsunobu reaction between $N^{2}, N^{2}$-bis $(t e r t-$ butyloxycarbonyl)-6-chloro-guanine and 3,5-O-dibenzyl-1-deoxy- $L$-ribose afforded isonucleoside 6. Finally, iso- $L$-guanosine was synthesized in 9 steps with $37.3 \%$ overall yield. Adopting Mitsunobu reaction as the key step, it has the merits of high steroseletivity and regioselectivity, mild reaction condition, and high yield. Currently developed approach could be used as a general synthetic strategy for the synthesis other related guanine isonucleosides.
\end{abstract}

Keywords nucleosides; total synthesis; glycosidation; Mitsunobu reaction; isonucleosides

核苷是 DNA 和 RNA 的基本结构单元, 长期以来对 核甘进行结构修饰以期发现新的抗癌和抗病毒药物是 新药研发的热点之一 ${ }^{[1]}$. 迄今为止, 根据美国食品与药 品管理局(FDA)统计, 上市的 60 多个抗肿瘤药物中有 9 个 $(15 \%)$ 是核苷及其类似物, 29 个抗病毒药物中有 16 个 $(55 \%)$ 是核苷及其类似物. 针对核苷化合物在核苷水解 酶作用下易于发生降解的潜在药物代谢问题, Montgomery 等 ${ }^{[2]}$ 在 20 世纪 70 年代末首次提出了异核苷的概 念(Isomeric nucleoside 或者 Isonucleoside), 主要是指核 苷碱基从核糖呋喃环的 1 '位移动到糖环的其他位置(例 如 2'或者 3'等). 由于碱基位置的移动, 新构建的 $\mathrm{C}-\mathrm{N}$ 键在核苷水解酶的作用下非常稳定. 在过去的三十年 里, 异核苷作为一类新的抗肿瘤和抗病毒药物先导化合
物得到了广泛的研究, 药物化学家合成出了大量的异核 苷化合物, 主要的研究小组有 $\mathrm{Nair}^{[3]} 、$ Matsuda $^{[4]}$ 、张礼 和 ${ }^{[5]}$ 和我们小组 ${ }^{[6]}$ 等, 可惜的是目前仍未篮选出能进入 临床使用的药物分子.

2013 年, 杨振军小组首次研究发现异核苷 $(D$-或者 $L$-胸腺嘧啶) 嵌杂的寡聚核苷酸在干扰核糖核酸 $(\operatorname{siRNA})^{[7]}$ 和适配体(Aptamer) ${ }^{[8]}$ 中表现出优良的生物学 效应; 进一步的研究表明异核苷修饰的血红蛋白结合适 配体(TBA)能够增强与血红蛋白的亲和力, 从而增强凝 血效果 ${ }^{[9]}$, 另一方面异核苷修饰的适配体能够显著增加 对杂核核糖蛋白 A1 (heterogeneous nuclear ribonucleoprotein A1, hnRNP A1)的亲和力，从而具有潜在的抑制 肿瘤形成作用 ${ }^{[10]}$, 为异核苷的研究和应用开辟了新的

\footnotetext{
* Corresponding authors. E-mail: xiaoqiang@tsinghua.org.cn; dinghaixin@163.com Received January 27, 2019; revised March 18, 2019; published online April 16, 2019.

Project supported by the National Natural Science Foundation of China (Nos. 21462019, 21676131), the Bureau of Science \& Technology of Jiangxi Province (No. 20143ACB20012) and the Jiangxi Science \& Technology Normal University (Doctor Startup Fund No. 2018BSQD022).

国家自然科学基金(Nos. 21462019, 21676131)、江西省科技厅重点(No. 20143ACB20012)和江西科技师范大学博士启动基金(No. 2018BSQD022)资助项 目.
} 
思路. 为了研究 $L$-鸟嘌呤异核苷嵌杂的寡聚核苷酸的生 物学性质, 首先需要在克级规模对 $L$-鸟嘌呤异核苷进行 合成. 在文献中, 张礼和小组报道 ${ }^{[1]} 了$ 了 $D$-木糖为起始 原料, 首先经过 5 步反应构建关键中间体环氧化合物 $\mathbf{2}$, 然后通过鸟嘌呤碱基 $\mathbf{3}$ 对环氧化合物 $\mathbf{2}$ 的开环反应构建 异核苷 $\mathrm{C}-\mathrm{N}$ 键(Scheme 1a). 在合成中, 由于鸟嘌呤碱 基 3 在有机溶剂中的溶解度非常差, 同时由于化合物 $\mathbf{2}$ 中环氧结构稳定很高, 在关键步骤中需要使用 $N, N$ 二甲 基甲酰胺(DMF)作为溶剂, 在 $120{ }^{\circ} \mathrm{C}$ 加热的条件下进行 核苷键的构建, 反应条件苛刻, 异核苷中间体 $\mathbf{4}$ 的产率 低于 $45 \%$. 该路线不可避免地产生 N-9 取代和 3'取代副 产物, 分离困难, 同时未见 $L$-鸟嘌呤异核苷 1 的详细表 征数据报道. 本文将报道以 $L$-核糖为起始原料进行 $L-$ 鸟嘌呤异核苷 1 的合成工艺研究(Scheme 1b).

\section{1 结果与讨论}

首先在 $1 \%$ 的氯化氢甲醇溶液中将 $L$-核糖高产率 转化为 $L$-呋喃核糖甲苷, 减压除去溶剂后, 产物不经分 离纯化, 直接在醋酸钠和醋䣶体系中加热回流, 将剩余 着基进行乙酰化, 然后在醋酐和醋酸混合溶剂中将甲苷 在浓硫酸催化下转化为相应的乙酰酯, 经过柱层析分离 纯化得到四乙酰化- $L$-呋喃核糖 $\boldsymbol{8}^{[12]}(\alpha: \beta=1: 5.3)$, 三 步总收率为 $82.0 \%$, 同时首次得到了 $\beta$ 构型的单晶衍射 晶体结构(如 Scheme 2 所示 $)^{[13]}$, 该方法步骤操作简便、 重现性好, 可以在 100 克级规模上进行. 然后四乙酰呋 喃核糖 8 在干燥的丙酮溶剂中, 以单质碘 $\left(\mathrm{I}_{2}\right)$ 为催化剂,
以 $82 \%$ 产率得到丙叉保护的核糖 $\mathbf{9}^{[14]}$, 之后在甲醇溶液 中, 以碳酸钾为碱，几乎定量地脱除乙酰基得到丙叉保 护的核糖 10. 接着以 $\mathrm{DMF}$ 为溶剂, 以氢化钠 $(\mathrm{NaH})$ 为 碱，用苄溴保护核糖的 3,5 位羟基，同样几乎定量得到 糖 11. 最后用三氟甲磺酸三甲基硅酯(TMSOTf)为路易 斯酸, 用三乙基硅氢 $\left(\mathrm{Et}_{3} \mathrm{SiH}\right)$ 还原原位形成的端头碳正 离子, 在得到关键中体 $\mathbf{5}$ 的同时, 不可避免地会产生异 丙醚产物 $\mathbf{1 2}^{[15]}$. 该异丙醚副产物 12 为热力学产物. 通 过条件优化, 发现降低反应的浓度 $(0.09 \mathrm{mmol} / \mathrm{mL})$ 和降 低反应温度，可以有效降低副产物的形成，最后以 $82 \%$ 产率得到关键糖中间体 $\mathbf{5}$.

在得到关键糖中间体 $\mathbf{5}$ 后，我们尝试将 2 位羟基转 换为不同的离去基团 ${ }^{[16]}$, 例如甲磺酸酯、对甲苯磺酸酯 和三氟甲磺酸酯等, 然后与碱基 13 进行取代反应合成 目标异核苷化合物，经过长时间的尝试，发现底物容易 发生消除反应, 产率很低 $(<20 \%)$, 同时伴随着 $N-7$ 副产 物的形成, 分离非常困难, 因此只能寻求别的合成途径.

近些年来, Mitsunobu 反应在核苷化学中得到了广 泛的应用 ${ }^{[17]}$, 该反应条件温和, 对手性羟基立体专一性 发生构型的翻转, 因此尝试着用该反应来解决我们面临 的问题. 由于 6-氯鸟嘌呤在有机溶剂中的溶解度特别 差，同时 2-氨基由于带有活泼氢，可能会和糖中间体 $\mathbf{5}$ 中的 2 位羟基发生 Mitsunobu 反应，因此首先需要将 2 位氨基进行保护. 选择近年来在核苷碱基保护中得到广 泛应用的叔丁氧羰基(Boc)来实现目的. 通过对文献报
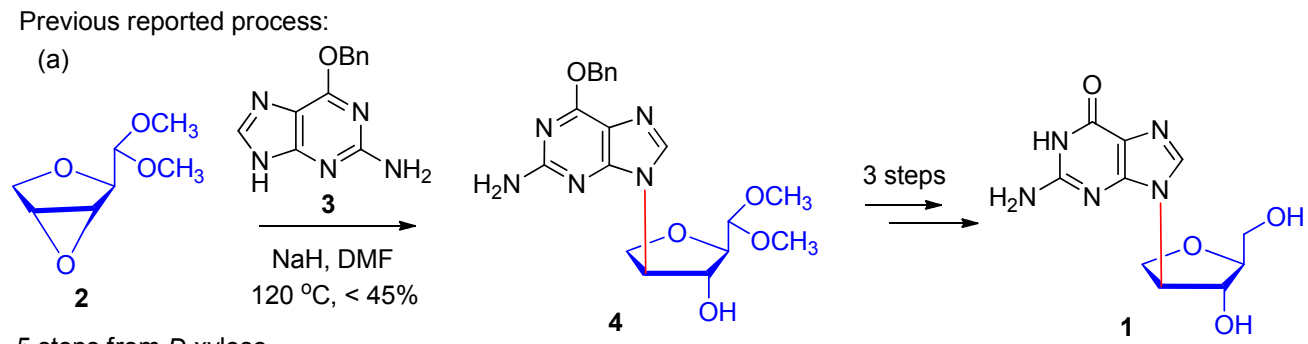

5 steps from $D$-xylose

Current developed process:

(b)

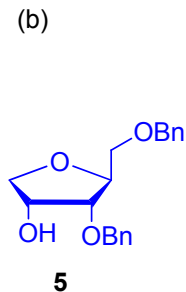

5 steps from $L$-ribose with $51.9 \%$ yield

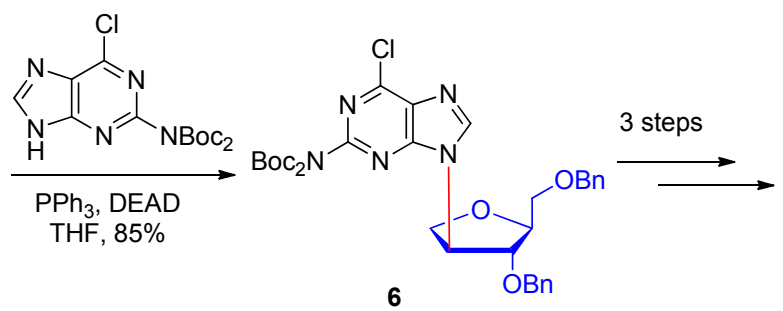

6

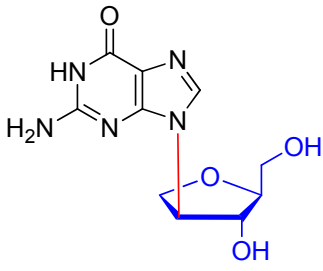

$37.3 \%$ overal yield 1

图式 $1 L$-鸟嘌呤异核苷的合成策略

Scheme 1 Synthetic strategies of iso-L-guanosine 
<smiles>OCC1O[C@@H](O)[C@@H](O)[C@@H]1O</smiles>

(a) $\mathrm{CH}_{3} \mathrm{OH}, \mathrm{CH}_{3} \mathrm{COCl}$ (b) $\mathrm{Ac}_{2} \mathrm{O}, \mathrm{AcONa}, 70^{\circ} \mathrm{C}$

(c) $\mathrm{AcOH}, \mathrm{Ac}_{2} \mathrm{O}, \mathrm{H}_{2} \mathrm{SO}_{4}$ $50{ }^{\circ} \mathrm{C}, 82 \%$

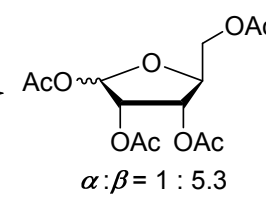

8

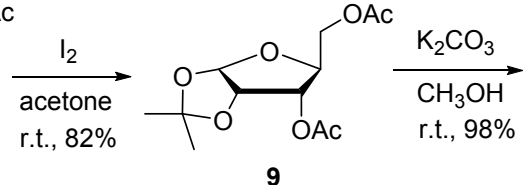

9

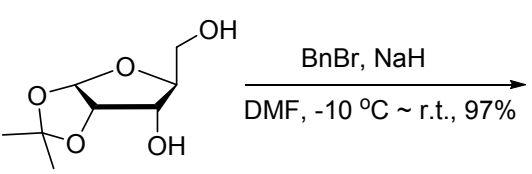

10<smiles>CC1(C)OC2OC(CO[18OH])C(O)C2O1</smiles>

11

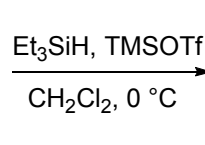

(n)

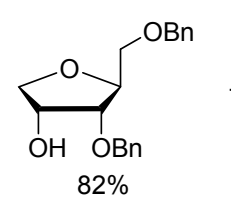

5

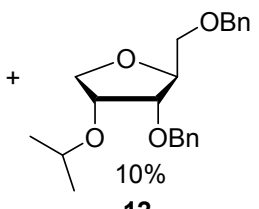

12<smiles>Nc1nc(Cl)c2nc[nH]c2n1</smiles>

13

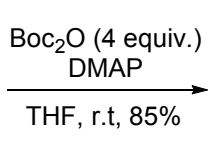
DMAP<smiles>CC(C)(C)OC(=O)c1nc(Cl)c2nc[nH]c2n1</smiles>

14

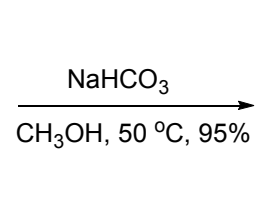<smiles>CC(C)(C)OC(C)(C)Oc1nc(Cl)c2nc[nH]c2n1</smiles>

15

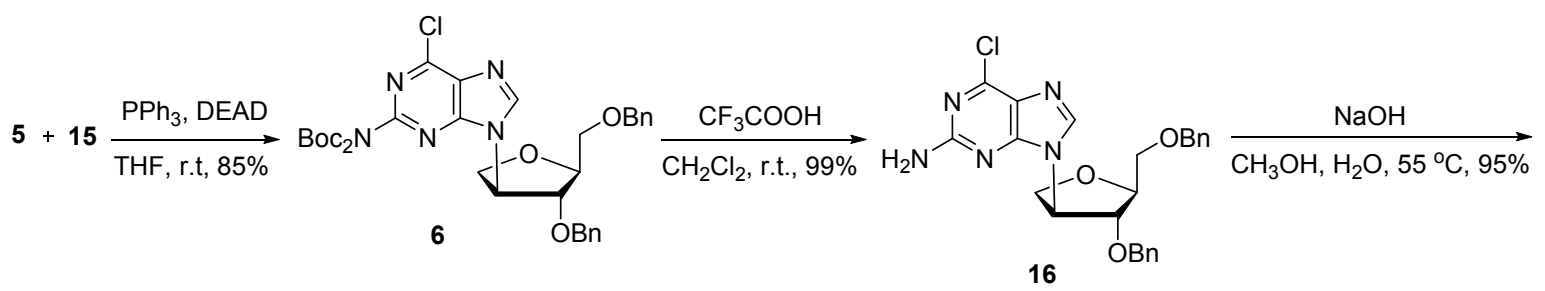<smiles>Nc1nc2c(ncn2C2COCC2CO)c(=O)[nH]1</smiles>

17

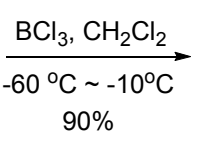
$90 \%$

图式 $2 L$-鸟嘌呤异核苷 1 的全合成

Scheme 2 Total synthesis of $i s o$ - $L$-guanosine
道 ${ }^{[18]}$ 的条件进行摸索和改进, 首先在 4 equiv. $\mathrm{Boc}_{2} \mathrm{O}$ 作 用下, 对环外氨基和 $N-9$ 氨基进行全保护, 然后将得到 的产物溶解在甲醇中, 用弱碱碳酸氢钠溶液水解去除 $N-9$ 氨基上的 Boc 基团, 从而以 $81 \%$ 的总收率得到环外 氨基双 Boc 保护的碱基 15, 改进后的步骤在 100 克规模 上顺利重复来合成碱基 $\mathbf{1 5}$.

接着尝试用 Mitsunobu 反应来合成异核苷 $\mathbf{6}$, 经过 条件优化, 以四氢呋喃(THF)为溶剂, 在三苯基膦 $\left(\mathrm{PPh}_{3}\right)$ 和偶氮二甲酸二乙酯(DEAD)作用下, 碱基 15 和糖中间 体 5 可以顺利发生缩合反应得到异核苷 $\mathbf{6}$, 同时 HPLC 分析未发现碱基 N-7 位偶联副产物的生成. 然后尝试在 强碱的作用下, 一步水解异核苷 6 的 6 位氯原子和 Boc 保护基团, 但是反应产物复杂, 很难进行分离. 之后, 改变反应策略, 首先在三氟乙酸作用下几乎定量水解脱 除 Boc 保护基团, 再在 $12.5 \mathrm{~mol} / \mathrm{L}$ 氢氧化钠溶液中回流 水解去除 6 位的氯原子, 顺利以 $94 \%$ 产率得到异核甘 17. 接着, 首先尝试用 $\mathrm{Pd} / \mathrm{C}$ 加氢的方法脱除苠基, 但是 由于氨基的存在, 反应非常慢, 不能反应完全. 最后,
采用二氯甲烷 $(\mathrm{DCM})$ 为溶剂, 低温下 $\left(-60{ }^{\circ} \mathrm{C}\right)$ 用三氯化 硼 $\left(\mathrm{BCl}_{3}\right)$ 可以顺利脱除苄基 ${ }^{[19]}$, 以 $90 \%$ 产率得到目标分 子 $L$-鸟嘌呤异核苷 1, HPLC 分析显示纯度大于 $98.5 \%$. 文献报道 $L$-鸟嘌呤异核苷 1 数据表征不够详细, 本文所 有报道合成中间体结构都经严格的光谱数据确证.

\section{2 结论}

以 $L$-核糖为原料, 以 Mitsunobu 反应进行碱基 $\mathbf{1 5}$ 和糖中间体 $\mathbf{5}$ 的缩合反应合成异核苷 $\mathbf{6}$ 为关键步骤, 经 过 9 步反应，以 $37.3 \%$ 的总产率完成了 $L$-鸟嘌呤异核苷 1 的全合成研究. 研究表明 Mitsunobu 反应可以显著提 高核苷键生成的产率和避免副反应的发生. 该全合成路 线反应条件温和、反应中间体容易纯化、总产率高、重 现性好, 可以作为鸟嘌呤异核苷的通用合成路线. $L$-鸟 嘌呤异核苷 1 经过标准的三步反应就可以得到 DNA 合成单体亚磷酰胺, 嵌入寡聚核苷酸中进行相关的生物 学性质研究正在进行中. 


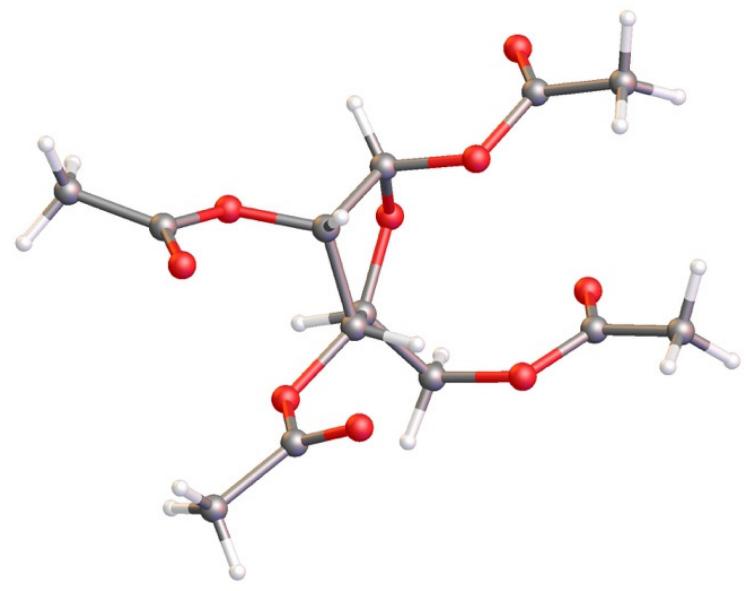

图 2 1,2,3,4-四- $O$-乙酰基- $\beta$ - $L$-核糖(8) 的单晶衍射结构 Figure 2 X-ray crystal structure of 1,2,3,4-tetra- $O$-acetyl- $\beta$ - $L$ ribose (8)

\section{3 实验部分}

\section{1 仪器与试剂}

Bruker AV400 型核磁共振仪(德国 Bruker 公司); AB SCIEX Triple TOF 4600 型高分辨质谱仪(美国 AB SCIEX 公司); Autopol IV-T A21200-T 型全自动数字式旋 光仪(美国 Rudolph 公司); M-560 熔点测定仪(BUCHI 公 司). 无水二氯甲烷 $\left(\mathrm{CH}_{2} \mathrm{Cl}_{2}\right)$ 相应的分析纯试剂加入氢化 钙回流 $3 \mathrm{~h}$, 使用前蒸出; 无水四氢呋喃(THF)为分析纯, 加入四氢铝锂回流 $3 \mathrm{~h}$, 使用前蒸出; 无水甲醇 $\left(\mathrm{CH}_{3} \mathrm{OH}\right)$ 为分析纯, 加入镁和碘回流 $3 \mathrm{~h}$, 蒸出后密封保存. 所用 常规试剂均购自北京伊诺凯科技有限公司.

\section{2 实验方法}

\subsubsection{1,2,3,5-四- $O$-乙酰基- $L$-核糖(8)的合成}

冰浴条件下, 向无水甲醇 $(70 \mathrm{~mL})$ 中缓慢加入乙酰 氯 $(5 \mathrm{~mL})$. 搅拌 $30 \mathrm{~min}$ 后, 分批加入化合物 $L$-核糖 7 $(5.0 \mathrm{~g}, 33.3 \mathrm{mmol})$, 加完后继续反应 $2 \mathrm{~h}$. 薄层色谱 (TLC) 检测显示反应完全后, 用氢氧化钠甲醇溶液 ( 0.1 $\mathrm{g} / \mathrm{m}$ ) 将反应液调至中性, 反应液减压浓缩得到淡黄色油 状化合物. 氩气保护下, 向该淡黄色油状化合物加入醋 酸酐(17.9 g, $175.5 \mathrm{mmol})$, 无水乙酸钠(14.3 g, 174.4 $\mathrm{mmol}), 70{ }^{\circ} \mathrm{C}$ 反应 $4 \mathrm{~h}$. TLC 检测显示反应完全后, 将反 应液倒入冰水 $(200 \mathrm{~mL})$ 中, 用二氯甲烷 $(200 \mathrm{~mL} \times 3)$ 萃 取, 有机相分别用水 $(100 \mathrm{~mL} \times 3)$ 、饱和碳酸氢钠溶液 $(150 \mathrm{~mL} \times 3)$ 、饱和食盐水 $(100 \mathrm{~mL} \times 2)$ 萃取和无水硫酸 镁干燥. 过滤液减压浓缩得到黄色油状物, 将其溶于冰 醋酸 $(100 \mathrm{~mL})$, 加入醋酸䣲 $(5.0 \mathrm{~g}, 49.0 \mathrm{mmol})$, 冰浴条件 下加入 $2 \sim 3$ 滴浓硫酸, 反应体系升温至 $50{ }^{\circ} \mathrm{C}$ 反应 $4 \mathrm{~h}$, $\mathrm{TLC}$ 检测显示反应完全后, 将反应液倒入冰水 $(200 \mathrm{~mL})$ 中, 加入二氯甲烷 $(200 \mathrm{~mL} \times 3)$ 溶解, 有机相分别用水 $(100 \mathrm{~mL} \times 3)$ 、饱和碳酸氢钠溶液 $(150 \mathrm{~mL} \times 3)$ 、饱和食
盐水 $(100 \mathrm{~mL} \times 2)$ 洗涤、无水硫酸镁干燥. 过滤液减压浓 缩得粗产物, 通过硅胶柱层析分离纯化得白色固体 $\mathbf{8} \boldsymbol{\beta}$ 和 $8 \alpha(10.0 \mathrm{~g}, \alpha: \beta=1: 5.3)$, 产率为 $82 \%$. 8 $\beta$ : m.p. 81 $82{ }^{\circ} \mathrm{C}$ (文献值 ${ }^{[12(b)]}: 80 \sim 83{ }^{\circ} \mathrm{C}$ ); $[\alpha]_{\mathrm{D}}^{25}+13.0$ (c 0.10, $\left.\mathrm{CH}_{3} \mathrm{OH}\right) ;{ }^{1} \mathrm{H}$ NMR $\left(400 \mathrm{MHz}\right.$, DMSO- $\left.d_{6}\right) \delta: 6.02(\mathrm{~s}, 1 \mathrm{H})$, $5.24(\mathrm{~d}, J=1.9 \mathrm{~Hz}, 2 \mathrm{H}), 4.32$ (s, 1H), 4.29 (d, $J=3.1 \mathrm{~Hz}$, 1H), 4.06 (q, $J=5.5 \mathrm{~Hz}, 1 \mathrm{H}), 2.09$ (s, 3H), 2.07 (s, 3H), 2.04 (s, 3H), 2.04 (s, 3H); ${ }^{13} \mathrm{C}$ NMR (101 MHz, DMSO- $d_{6}$ ) $\delta: 170.5,170.0,169.8,169.3,98.1,79.5,74.0,70.5,63.5$, 21.3, 21.0, 20.8, 20.7; HRMS calcd for $\mathrm{C}_{13} \mathrm{H}_{18} \mathrm{O}_{9} \mathrm{Na}[\mathrm{M}+$ $\mathrm{Na}]^{+} 341.0843$, found 341.0840. 8 $\alpha$ : 油状物, $[\alpha]_{\mathrm{D}}^{25}-$ 42.6 (c $0.05, \mathrm{CH}_{3} \mathrm{OH}$ ); ${ }^{1} \mathrm{H}$ NMR (400 MHz, DMSO- $d_{6}$ ) $\delta$ : $6.31(\mathrm{~d}, J=4.5 \mathrm{~Hz}, 1 \mathrm{H}), 5.27 \sim 5.15(\mathrm{~m}, 1 \mathrm{H}), 4.38(\mathrm{q}, J=$ $3.5 \mathrm{~Hz}, 1 \mathrm{H}), 4.24(\mathrm{dd}, J=12.0,3.0 \mathrm{~Hz}, 1 \mathrm{H}), 4.13(\mathrm{dd}, J=$ 12.0, 3.0 Hz, 1H), 2.08 (s, 3H), 2.07 (s, 3H), 2.04 (s, 3H), 2.03 (s, $3 \mathrm{H}$ ); ${ }^{13} \mathrm{C}$ NMR (101 MHz, DMSO- $\left.d_{6}\right) \delta: 170.5$, 170.2, 169.9, 169.6, 94.1, 81.1, 69.9, 69.7, 63.5, 21.2, 21.0, 20.8, 20.5; HRMS calcd for $\mathrm{C}_{13} \mathrm{H}_{18} \mathrm{O}_{9} \mathrm{Na}[\mathrm{M}+\mathrm{Na}]^{+}$ 341.0843 , found 341.0841 .

3.2.2 3,5-O-二乙酰基-1,2- $O$ - 亚异丙基- $L$-核糖(9)的 合成

氩气保护下将化合物 $8 \boldsymbol{\beta}(5.0 \mathrm{~g}, 15.7 \mathrm{mmol})$ 溶于丙 酮, 加入催化量的碘 $(0.2 \mathrm{~g}, 0.8 \mathrm{mmol})$. 反应体系室温下 反应 $3.5 \mathrm{~h}, \mathrm{TLC}$ 检测显示反应完全后, 加入饱和硫代硫 酸钠水溶液 $(25 \mathrm{~mL})$ 淬灭反应. 反应液减压浓缩蒸去丙 酮溶剂, 浓缩物加入水 $(100 \mathrm{~mL})$ 稀释, 用二氯甲烷 $(100$ $\mathrm{mL} \times 3)$ 萃取, 分别用水 $(100 \mathrm{~mL} \times 3)$ 、饱和碳酸氢钠溶液 $(100 \mathrm{~mL} \times 3)$ 、饱和食盐水 $(100 \mathrm{~mL} \times 2)$ 洗涤、无水硫酸 镁干燥. 过滤液减压浓缩得粗产物, 通过进一步硅胶柱 层析分离纯化得黄色油状物 9 (3.5 g), 产率为 $82 \%$. $[\alpha]_{\mathrm{D}}^{25}-125.9$ (c $\left.0.11, \mathrm{CH}_{3} \mathrm{OH}\right) ;{ }^{1} \mathrm{H}$ NMR $(400 \mathrm{MHz}$, DMSO- $\left.d_{6}\right) \delta: 5.81(\mathrm{~d}, J=3.6 \mathrm{~Hz}, 1 \mathrm{H}), 4.77(\mathrm{t}, J=4.2 \mathrm{~Hz}$, $1 \mathrm{H}), 4.76 \sim 4.61(\mathrm{~m}, 1 \mathrm{H}), 4.24(\mathrm{~d}, J=13.0 \mathrm{~Hz}, 1 \mathrm{H}), 4.18$ (q, $J=5.6 \mathrm{~Hz}, 1 \mathrm{H}), 4.05(\mathrm{dd}, J=12.2,5.4 \mathrm{~Hz}, 1 \mathrm{H}), 2.06(\mathrm{~s}$, $3 \mathrm{H}), 2.03(\mathrm{~s}, 3 \mathrm{H}), 1.45$ (s, 3H), $1.27(\mathrm{~s}, 3 \mathrm{H}) ;{ }^{13} \mathrm{C} \mathrm{NMR}$ $\left(101 \mathrm{MHz}\right.$, DMSO- $\left.d_{6}\right) \delta: 170.6,170.2,112.7,104.4,77.3$, 75.6, 72.4 , 62.9, 26.9, 26.8, 21.0, 20.9; HRMS calcd for $\mathrm{C}_{12} \mathrm{H}_{18} \mathrm{O}_{7} \mathrm{Na}[\mathrm{M}+\mathrm{Na}]^{+}$297.0944, found 297.0942 .

\subsubsection{1,2-O-亚异丙基- $L$ - 核糖(10)的合成}

将化合物 $9(5.0 \mathrm{~g}, 18.2 \mathrm{mmol})$ 溶于甲醇 $(40 \mathrm{~mL})$ 中, 加入无水碳酸钾 $(3.0 \mathrm{~g}, 21.7 \mathrm{mmol})$, 室温下搅拌 $2 \mathrm{~h}$. TLC 检测显示反应完全后, 反应液减压旋干, 粗产物通 过柱层析分离纯化得白色固体 10 (3.4 g), 产率为 98\%. m.p. $87 \sim 88{ }^{\circ} \mathrm{C}$ (文献值 ${ }^{[20]}: 88 \sim 89{ }^{\circ} \mathrm{C}$ ); $[\alpha]_{\mathrm{D}}^{25}-68.5(c$ $\left.0.10, \mathrm{CH}_{3} \mathrm{OH}\right) ;{ }^{1} \mathrm{H}$ NMR $\left(400 \mathrm{MHz}\right.$, DMSO- $\left.d_{6}\right) \delta: 5.65(\mathrm{~d}$, 
$J=3.6 \mathrm{~Hz}, 1 \mathrm{H}), 5.00(\mathrm{~d}, J=6.5 \mathrm{~Hz}, 1 \mathrm{H}), 4.65(\mathrm{t}, J=5.7$ $\mathrm{Hz}, 1 \mathrm{H}), 4.43(\mathrm{t}, J=3.9 \mathrm{~Hz}, 1 \mathrm{H}), 3.73 \sim 3.66(\mathrm{~m}, 2 \mathrm{H})$, $3.66 \sim 3.59(\mathrm{~m}, 1 \mathrm{H}), 3.42 \sim 3.35(\mathrm{~m}, 1 \mathrm{H}), 1.43(\mathrm{~s}, 3 \mathrm{H})$, $1.26(\mathrm{~s}, 3 \mathrm{H}) ;{ }^{13} \mathrm{C}$ NMR (101 MHz, DMSO- $\left.d_{6}\right) \delta: 111.6$, 103.8, 80.8, 79.6, 71.0, 60.7, 27.1, 26.9; HRMS calcd for $\mathrm{C}_{8} \mathrm{H}_{14} \mathrm{O}_{5} \mathrm{Na}[\mathrm{M}+\mathrm{Na}]^{+}$213.0733, found 213.0739

3.2.4 3,5-O-二華基-1,2- $O$-亚异丙基- $L$-核糖(11)的合 成

将化合物 10 (5.0 g, $26.3 \mathrm{mmol})$ 溶于无水 DMF (50 $\mathrm{mL})$ 中, 在 $-10{ }^{\circ} \mathrm{C}$ 低温下, 分批加入 $60 \% \mathrm{NaH}(4.2 \mathrm{~g}$, $175 \mathrm{mmol})$. 氩气保护下, 反应液搅拌 $1 \mathrm{~h}$ 后, 缓慢滴加 芐溴(18.0 g, $105.9 \mathrm{mmol})$. 滴加完毕, 继续反应 $1 \mathrm{~h}$ 后, 升温至室温反应 $4 \mathrm{~h}$. TLC 检测显示反应完全后, 加入冰 水 $(10 \mathrm{~mL})$ 淬灭反应, 将反应液倒入冰水 $(100 \mathrm{~mL})$ 中, 二 氯甲烷 $(200 \mathrm{~mL} \times 3)$ 萃取, 有机相分别用水 $(100 \mathrm{~mL} \times$ $3)$ 、饱和碳酸氢钠溶液 $(150 \mathrm{~mL} \times 3)$ 、饱和食盐水 $(100 \mathrm{~mL}$ $\times 2)$ 萃取, 无水硫酸镁干燥. 过滤液减压浓缩得到粗产 物, 通过硅胶柱层析分离纯化得淡黄色油状化合物 $\mathbf{1 1}$ $9.5 \mathrm{~g}$, 产率为 $97 \%$. $[\alpha]_{\mathrm{D}}^{25}-118.5\left(c 0.10, \mathrm{CH}_{3} \mathrm{OH}\right) ;{ }^{1} \mathrm{H}$ NMR (400 MHz, DMSO- $d_{6}$ ) $\delta: 7.7 \sim 7.16(\mathrm{~m}, 10 \mathrm{H}), 5.73$ (d, $J=3.5 \mathrm{~Hz}, 1 \mathrm{H}), 4.71(\mathrm{t}, J=3.8 \mathrm{~Hz}, 1 \mathrm{H}), 4.64(\mathrm{~d}, J=$ $11.9 \mathrm{~Hz}, 1 \mathrm{H}), 4.55 \sim 4.39(\mathrm{~m}, 3 \mathrm{H}), 3.99(\mathrm{dd}, J=8.6,4.7$ $\mathrm{Hz}, 1 \mathrm{H}), 3.74$ (dd, $J=9.1,4.2 \mathrm{~Hz}, 1 \mathrm{H}), 3.65$ (d, $J=11.0$ $\mathrm{Hz}, 1 \mathrm{H}$ ), 3.48 (dd, $J=11.2,5.0 \mathrm{~Hz}, 1 \mathrm{H}), 1.45$ (s, 3H), 1.29 $(\mathrm{s}, 3 \mathrm{H}) ;{ }^{13} \mathrm{C}$ NMR (101 MHz, DMSO- $\left.d_{6}\right) \delta: 138.7,138.4$, 128.7, 128.1, 128.0, 127.9, 112.2, 104.3, 77.9, 76.9, 72.8, 71.4, 69.3, 27.1, 27.0; HRMS calcd for $\mathrm{C}_{22} \mathrm{H}_{26} \mathrm{O}_{5} \mathrm{Na}[\mathrm{M}+$ $\mathrm{Na}]^{+}$393.1673, found 393.1673.

\subsubsection{3,5-O-二芐基-1-脱氧- $L$-核糖(5)的合成}

冰浴条件下, 将化合物 $\mathbf{1 1}(5.0 \mathrm{~g}, 13.5 \mathrm{mmol})$ 溶于无 水二氯甲烷 $(150 \mathrm{~mL})$ 中, 加入三乙基硅烷 $(7.9 \mathrm{~g}, 68.0$ $\mathrm{mmol})$ 后, 缓慢滴加 TMSOTf (4.6 g, $20.7 \mathrm{mmol})$. 滴加 完毕, 反应体系自然升温至室温反应 $3 \mathrm{~h}$. TLC 检测显 示反应完全后, 加入冰水 $(10 \mathrm{~mL})$ 淬灭反应, 将反应液 倒入冰水 $(100 \mathrm{~mL})$ 中, 二氯甲烷 $(100 \mathrm{~mL} \times 3)$ 萃取, 有机 相分别用水 $(100 \mathrm{~mL} \times 3)$ 、饱和碳酸氢钠溶液 $(150 \mathrm{~mL} \times$ $3)$ 、饱和食盐水 $(100 \mathrm{~mL} \times 2)$ 萃取, 无水硫酸镁干燥. 过 滤液减压浓缩得粗产物, 通过硅胶柱层析分离纯化得黄 色油状化合物 $53.5 \mathrm{~g}$ 和淡黄色油状化合物 $\mathbf{1 2} 0.5 \mathrm{~g}$.

化合物 5: 产率为 $82 \% .[\alpha]_{\mathrm{D}}^{25}-92.9$ (c 0.08 , $\mathrm{CH}_{3} \mathrm{OH}$ ); ${ }^{1} \mathrm{H}$ NMR $\left(400 \mathrm{MHz}\right.$, DMSO- $\left.d_{6}\right) \delta: 7.42 \sim 7.22$ $(\mathrm{m}, 10 \mathrm{H}), 4.88(\mathrm{~d}, J=5.1 \mathrm{~Hz}, 1 \mathrm{H}), 4.69(\mathrm{~d}, J=12.0 \mathrm{~Hz}$, $1 \mathrm{H}), 4.57 \sim 4.40(\mathrm{~m}, 3 \mathrm{H}), 4.22(\mathrm{~d}, J=3.8 \mathrm{~Hz}, 1 \mathrm{H}), 3.98 \sim$ $3.82(\mathrm{~m}, 2 \mathrm{H}), 3.80 \sim 3.68(\mathrm{~m}, 1 \mathrm{H}), 3.64 \sim 3.57(\mathrm{~m}, 1 \mathrm{H})$, $3.57 \sim 3.50(\mathrm{~m}, 1 \mathrm{H}), 3.49 \sim 3.40(\mathrm{~m}, 1 \mathrm{H}) ;{ }^{13} \mathrm{C}$ NMR $(101$
MHz, DMSO- $\left.d_{6}\right) \delta$ : $138.9,138.9,128.6,128.6,128.0$, $127.8,80.0,79.8,73.2,72.7,71.4,71.0,69.3$; HRMS calcd for $\mathrm{C}_{19} \mathrm{H}_{22} \mathrm{O}_{4} \mathrm{Na}[\mathrm{M}+\mathrm{Na}]^{+}$337.1410, found 337.1415.

1-脱氧-2- $O$-异丙基-3,5- $O$-二芐基- $L$-核糖(12): 产率 为 $10 \% .[\alpha]_{\mathrm{D}}^{25}-75.0\left(c \quad 0.11, \mathrm{CH}_{3} \mathrm{OH}\right) ;{ }^{1} \mathrm{H}$ NMR $(400$ MHz, DMSO- $\left.d_{6}\right) \delta: 7.43 \sim 7.18(\mathrm{~m}, 10 \mathrm{H}), 4.60(\mathrm{~d}, J=11.8$ $\mathrm{Hz}, 1 \mathrm{H}), 4.47 \sim 4.50(\mathrm{~m}, 3 \mathrm{H}), 4.11(\mathrm{~d}, J=4.7 \mathrm{~Hz}, 1 \mathrm{H})$, $3.97 \sim 3.91(\mathrm{~m}, 1 \mathrm{H}), 3.91 \sim 3.81(\mathrm{~m}, 2 \mathrm{H}), 3.71 \sim 3.60(\mathrm{~m}$, $2 \mathrm{H}), 3.53(\mathrm{dd}, J=10.7,3.4 \mathrm{~Hz}, 1 \mathrm{H}), 3.45(\mathrm{dd}, J=10.7,5.0$ $\mathrm{Hz}, 1 \mathrm{H}), 1.11(\mathrm{t}, J=6.5 \mathrm{~Hz}, 6 \mathrm{H}) ;{ }^{13} \mathrm{C}$ NMR $(101 \mathrm{MHz}$, DMSO- $\left.d_{6}\right) \delta: 138.9,138.8,128.7,128.6,128.1,127.9$, $127.8,80.5,78.9,74.9,72.8,71.5,71.1,70.9,70.7,23.0$, 22.7; HRMS calcd for $\mathrm{C}_{22} \mathrm{H}_{28} \mathrm{O}_{4} \mathrm{Na}[\mathrm{M}+\mathrm{Na}]^{+} 379.1879$, found 379.1873 .

3.2.6 $N^{2}, N^{2}, N^{9}$-三叔丁氧羰基-6-氯乌嘌呤(14)的合成

氩气保护下, 将 6-氯鸟嘌呤 $\mathbf{1 3}(8.5 \mathrm{~g}, 50.0 \mathrm{mmol})$ 和 DMAP $(0.6 \mathrm{~g}, 4.9 \mathrm{mmol})$ 溶于无水四氢呋喃 $(100 \mathrm{~mL})$ 中, 冰浴下缓慢滴加 $\mathrm{Boc}_{2} \mathrm{O}(43.6 \mathrm{~g}, 0.2 \mathrm{~mol})$. 滴加完毕, 反应体系升温至室温继续反应 $8 \mathrm{~h}$. TLC 检测显示反应 完全后, 反应液减压旋干得粗产物, 通过硅胶柱层析分 离纯化得白色固体 14 (20.0 g), 产率为 85\%. m.p. 50 $52{ }^{\circ} \mathrm{C}$ (文献值 ${ }^{[18 \mathrm{~d}]}$ : $50 \sim 51{ }^{\circ} \mathrm{C}$ ); ${ }^{1} \mathrm{H}$ NMR $(400 \mathrm{MHz}$, DMSO- $\left.d_{6}\right) \delta$ : $9.01(\mathrm{~s}, 1 \mathrm{H}), 1.62(\mathrm{~s}, 9 \mathrm{H}), 1.42(\mathrm{~s}, 18 \mathrm{H}) ;{ }^{13} \mathrm{C}$ NMR (101 MHz, DMSO- $\left.d_{6}\right) \delta$ : 152.8, 152.3, 150.7, 150.3, 147.5, 145.5, 130.7, 87.2, 84.0, 27.9, 27.8; HRMS calcd for $\mathrm{C}_{20} \mathrm{H}_{28} \mathrm{ClN}_{5} \mathrm{O}_{6} \mathrm{Na}[\mathrm{M}+\mathrm{Na}]^{+} 492.1620$, found 492.1623 .

\subsection{7 $N^{2}, N^{2}$-二叔丁氧羰基-6-氯鸟嘌呤 $(\mathbf{1 5})$ 的合成}

将化合物 14 (4.0 g, $8.5 \mathrm{mmol}$ )溶于甲醇 $(100 \mathrm{~mL})$ 中, 加入饱和碳酸氢钠溶液 $(25 \mathrm{~mL}), 50{ }^{\circ} \mathrm{C}$ 反应 $1.5 \mathrm{~h}$. TLC 检 测显示完全反应后，低温旋干甲醇溶剂，用 $5 \mathrm{~mol} / \mathrm{L}$ 盐 酸调至中性, 析出白色固体, 过滤得化合物 $153.0 \mathrm{~g}$, 产 率为 $95 \%$. m.p. $91 \sim 93{ }^{\circ} \mathrm{C} ;{ }^{1} \mathrm{H}$ NMR $(400 \mathrm{MHz}$, DMSO- $\left.d_{6}\right) \delta: 8.24$ (s, $\left.1 \mathrm{H}\right), 1.38(\mathrm{~s}, 18 \mathrm{H}) ;{ }^{13} \mathrm{C}$ NMR $(101$ MHz, DMSO- $\left.d_{6}\right) \delta$ : $162.6,156.1,151.6,148.2,145.0$, 131.1, 82.7, 28.0; HRMS calcd for $\mathrm{C}_{15} \mathrm{H}_{20} \mathrm{ClN}_{5} \mathrm{ONa}[\mathrm{M}+$ $\mathrm{Na}]^{+}$392.1096, found 392.1083.

3.2.8 $N^{2}, N^{2}$-二叔丁氧羰基-6-氯鸟嘌呤- $3^{\prime}, 5^{\prime}-O$-二芳 基-1'-脱氧- $2^{\prime}-L$-异核苷(6) 的合成

氩气保护下, 将糖 $5(5.0 \mathrm{~g}, 15.9 \mathrm{mmol})$ 、碱基 $\mathbf{1 5}$ (6.5 $\mathrm{g}, 17.6 \mathrm{mmol})$ 、三苯基膦 $(8.3 \mathrm{~g}, 31.6 \mathrm{mmol})$ 溶于无水四氢 呋喃中. 冰浴下反应液中缓慢滴加偶氮二甲酸二乙酯 (5.54 g, $31.8 \mathrm{mmol})$. 滴加完毕, 反应体系升温至室温, 继续反应 $10 \mathrm{~h} . \mathrm{TLC}$ 检测显示反应完全后, 减压旋干得 到粗产物, 通过硅胶柱层析分离纯化得黄色油状物 6 
$(9.0 \mathrm{~g})$, 产率为 $85 \% .[\alpha]_{\mathrm{D}}^{25}-69.2\left(c \quad 0.1, \mathrm{CH}_{3} \mathrm{OH}\right) ;{ }^{1} \mathrm{H}$ NMR ( $\left.400 \mathrm{MHz}, \mathrm{CDCl}_{3}\right) \delta: 8.37(\mathrm{~s}, 1 \mathrm{H}), 7.31 \sim 7.03(\mathrm{~m}$, $10 \mathrm{H}), 5.16(\mathrm{~s}, 1 \mathrm{H}), 4.74(\mathrm{~d}, J=11.8 \mathrm{~Hz}, 1 \mathrm{H}), 4.48 \sim 4.29$ $(\mathrm{m}, 3 \mathrm{H}), 4.23(\mathrm{~d}, J=3.0 \mathrm{~Hz}, 2 \mathrm{H}), 4.07 \sim 3.94(\mathrm{~m}, 2 \mathrm{H})$, $3.63(\mathrm{dd}, J=10.7,2.7 \mathrm{~Hz}, 1 \mathrm{H}), 3.44(\mathrm{dd}, J=10.7,3.6 \mathrm{~Hz}$, $1 \mathrm{H}), 1.39(\mathrm{~s}, 18 \mathrm{H}) ;{ }^{13} \mathrm{C} \mathrm{NMR}\left(101 \mathrm{MHz}, \mathrm{CDCl}_{3}\right) \delta: 152.3$, $151.9,151.3,151.0,144.9,137.3,137.1,128.6,128.6$, $128.2,128.1,127.9,85.6,84.9,83.9,73.6,72.8,71.7,68.5$, 60.2, 28.1; HRMS calcd for $\mathrm{C}_{34} \mathrm{H}_{40} \mathrm{ClN}_{5} \mathrm{O}_{7} \mathrm{Na}[\mathrm{M}+\mathrm{Na}]^{+}$ 688.2508 , found 688.2510 .

3.2 .96 - 氯嘌呤- $3{ }^{\prime}, 5^{\prime}-O$-二芳基- $1^{\prime}-$ 脱氧 $-2^{\prime}-L$ - 异核苷 (16)的合成

将化合物 $6(5.0 \mathrm{~g}, 7.5 \mathrm{mmol})$ 溶于二氯甲烷 $(40 \mathrm{~mL})$ 中, 缓慢滴加三氟乙酸 $(10 \mathrm{~mL}, 131.6 \mathrm{mmol})$, 室温下反 应 $3 \mathrm{~h}$. TLC 检测显示反应完全后, 加入冰水 $(10 \mathrm{~mL})$ 淬 灭反应, 将反应液倒入冰水 $(100 \mathrm{~mL})$ 中, 二氯甲烷 $(100$ $\mathrm{mL} \times 3)$ 萃取, 有机相分别用水 $(100 \mathrm{~mL} \times 3) 、 1 \mathrm{~mol} / \mathrm{L}$ 氢氧 化钠溶液 $(150 \mathrm{~mL} \times 3)$ 、饱和食盐水 $(100 \mathrm{~mL} \times 2)$ 萃取, 无水硫酸镁干燥. 过滤液减压浓缩得到粗产物, 通过硅 胶柱层析分离纯化得淡黄色油状物 $163.5 \mathrm{~g}$, 产率为 99\%. $[\alpha]_{\mathrm{D}}^{25}-138.0\left(c 0.09, \mathrm{CH}_{3} \mathrm{OH}\right) ;{ }^{1} \mathrm{H}$ NMR $(400 \mathrm{MHz}$, DMSO- $\left.d_{6}\right) \delta: 8.02(\mathrm{~d}, J=8.1 \mathrm{~Hz}, 1 \mathrm{H}), 7.45 \sim 7.21(\mathrm{~m}, 8 \mathrm{H})$, 7.16 (d, $J=8.0 \mathrm{~Hz}, 2 \mathrm{H}), 6.98$ (s, 2H), 4.95 (s, 1H), 4.67 (d, $J=12.2 \mathrm{~Hz}, 2 \mathrm{H}), 4.44$ (s, 2H), 4.34 (d, $J=12.0 \mathrm{~Hz}, 1 \mathrm{H})$, $4.18 \sim 4.10$ (m, 2H), 3.98 (d, $J=4.0 \mathrm{~Hz}, 1 \mathrm{H}), 3.57$ (d, $J=$ $4.0 \mathrm{~Hz}, 2 \mathrm{H}) ;{ }^{13} \mathrm{C}$ NMR (101 MHz, DMSO- $\left.d_{6}\right) \delta: 160.1$, $154.2,149.9,141.2,138.5,138.2,128.6,128.1,128.0$, 127.9, 127.8, 123.8, 84.8, 83.9, 72.8, 71.8, 70.3, 69.8, 59.9; HRMS calcd for $\mathrm{C}_{24} \mathrm{H}_{24} \mathrm{ClN}_{5} \mathrm{O}_{3} \mathrm{Na}[\mathrm{M}+\mathrm{Na}]^{+} 488.1460$, found 488.1459 .

3.2.10 3',5'- $O$-二芳基- $1^{\prime}$ - 脱氧- 2 ' $-L$ - 异鸟苷(17)的合 成

将化合物 $\mathbf{1 6}(5.0 \mathrm{~g}, 10.7 \mathrm{mmol})$ 溶于甲醇 $(80 \mathrm{~mL})$ 中, 加入氢氧化钠水溶液 $(20 \mathrm{~mL}, 12.5 \mathrm{~mol} / \mathrm{L}), 55{ }^{\circ} \mathrm{C}$ 加热反 应 $3 \mathrm{~h}$. TLC 检测显示反应完全后, 减压浓缩除去甲醇, 将反应液倒入冰水 $(100 \mathrm{~mL})$ 中, 加入二氯甲烷 $(100 \mathrm{~mL}$ $\times 3)$ 溶解, 有机相分别用水 $(100 \mathrm{~mL} \times 3)$ 、饱和食盐水 $(100 \mathrm{~mL} \times 2)$ 萃取, 无水硫酸镁干燥. 过滤液减压浓缩得 到粗产物, 通过硅胶柱层析分离纯化得到白色固体 17 $4.8 \mathrm{~g}$, 产率为 $95 \%$. $[\alpha]_{\mathrm{D}}^{25}-97.6\left(c 0.09, \mathrm{CH}_{3} \mathrm{OH}\right)$; m.p. $212 \sim 214{ }^{\circ} \mathrm{C} ;{ }^{1} \mathrm{H}$ NMR $\left(400 \mathrm{MHz}\right.$, DMSO- $\left.d_{6}\right) \delta: 10.65$ (s, $1 \mathrm{H}), 7.62(\mathrm{~s}, 1 \mathrm{H}), 7.22 \sim 7.21(\mathrm{~m}, 10 \mathrm{H}), 6.48(\mathrm{~s}, 2 \mathrm{H}), 4.83$ (s, 1H), $4.65(\mathrm{~d}, J=11.4 \mathrm{~Hz}, 1 \mathrm{H}), 4.53(\mathrm{~d}, J=11.8 \mathrm{~Hz}$, 1H), 4.42 (s, 2H), 4.18 (d, $J=7.3 \mathrm{~Hz}, 1 \mathrm{H}$ ), 4.09 (d, $J=4.9$ $\mathrm{Hz}, 1 \mathrm{H}), 4.02$ (s, 1H), 3.93 (s, 1H), 3.53 (s, 2H); ${ }^{13} \mathrm{C}$ NMR $\left(101 \mathrm{MHz}\right.$, DMSO- $\left.d_{6}\right) \delta: 157.3,154.1,151.4,138.6,138.2$, 135.4, 128.7, 128.2, 128.1, 127.9, 116.9, 85.4, 83.8, 72.8, 71.7, 70.9, 70.0, 59.6; HRMS calcd for $\mathrm{C}_{24} \mathrm{H}_{25} \mathrm{~N}_{5} \mathrm{O}_{4} \mathrm{Na}$ $[\mathrm{M}+\mathrm{Na}]^{+} 470.1799$, found 470.1798 .

\subsubsection{1 '-脱氧-2'- $L$-异鸟苷(1)的合成}

氩气保护下, 将化合物 $\mathbf{1 7}(1.0 \mathrm{~g}, 2.3 \mathrm{mmol})$ 溶于 10 $\mathrm{mL}$ 无水二氯甲烷中. 在 $-60{ }^{\circ} \mathrm{C}$ 低温下, 缓慢加入浓度 $1 \mathrm{~mol} / \mathrm{L}$ 的三氯化嗍的二氯甲烷溶液 $(8.9 \mathrm{~mL}, 8.9 \mathrm{mmol})$. 反应 $3 \mathrm{~h}$ 后, 将温度升至 $-10{ }^{\circ} \mathrm{C}$ 继续反应 $6 \mathrm{~h}$. TLC 检测 显示反应完全后，向反应液加入 $2 \mathrm{~mol} / \mathrm{L}$ 氢氧化钠溶液 $(5 \mathrm{~mL})$, 室温摚拌 $5 \mathrm{~h}$. 反应液减压旋干, 粗产物通过硅 胶柱层析分离纯化得 $0.5 \mathrm{~g}$ 白色固体 1, 产率为 $90 \%$. $[\alpha]_{\mathrm{D}}^{25}-12.8\left(c 0.03, \mathrm{CH}_{3} \mathrm{COOH}\right) ;$ m.p. $276 \sim 278{ }^{\circ} \mathrm{C} ;{ }^{1} \mathrm{H}$ NMR (400 MHz, DMSO- $d_{6}$ ) $\delta: 10.81$ (brs, $\left.1 \mathrm{H}, \mathrm{NH}\right), 7.76$ (s, 1H, H-8), 6.64 (s, 2H, NH $)_{2}, 5.76(\mathrm{~s}, 1 \mathrm{H}, \mathrm{OH}), 4.94$ (s, $1 \mathrm{H}, \mathrm{OH}), 4.65$ (d, $\left.J=4.4 \mathrm{~Hz}, 1 \mathrm{H}, \mathrm{H}-2^{\prime}\right), 4.28$ (s, 1H, H-4'), $4.21 \sim 4.02\left(\mathrm{~m}, 1 \mathrm{H}, \mathrm{H}-1^{\prime}\right), 3.92$ (dd, $J=9.2,4.5 \mathrm{~Hz}, 1 \mathrm{H}$, H-1'), 3.74 3.49 (m, 3H, H-3', H-5'); ${ }^{13} \mathrm{C}$ NMR (101 MHz, DMSO- $\left.d_{6}\right) \delta: 157.5$ (C-6), 154.2 (C-2), 151.6 (C-4), 135.7 (CH-8), 116.8 (C-5), 86.4 (CH-3'), 76.4 (CH-4'), $70.5\left(\mathrm{CH}_{2}-1^{\prime}\right), 61.9\left(\mathrm{CH}-2^{\prime}\right), 61.3\left(\mathrm{CH}_{2}-5^{\prime}\right)$; HRMS calcd for $\mathrm{C}_{10} \mathrm{H}_{13} \mathrm{~N}_{5} \mathrm{O}_{4} \mathrm{Na}[\mathrm{M}+\mathrm{Na}]^{+}$290.0860, found 290.0865 .

辅助材料(Supporting Information) 化合物 1 和 7 17 的核磁共振谱图. 这些材料可以免费从本刊网站 (http://sioc-journal.cn/)上下载.

\section{References}

[1] (a) Yates, M. K.; Seley-Radtke, K. L. Antiviral Res. 2019, 162, 5. (b) Seley-Radtke, K. L.; Yates, M. K. Antiviral Res. 2018, 154, 66. (c) Jordheim, L. P.; Durantel, D.; Zoulim, F.; Dumontet, C. Nat. Rev. Drug Discovery 2013, 12, 447.

(d) Zhou, X.-X.; Littler, E. Curr. Top. Med. Chem. 2006, 6, 851. (e) Fan, X.-S.; Zhang, X.-Y.; Wang, X.; Qu, G.-R. Chin. J. Org. Chem. 2008, 28, 1888 (in Chinese). (范学森, 张新迎, 王霞, 渠桂荣, 有机化学, 2008, 28, 1888.)

[2] (a) Montgomery, J. A.; Clayton, S. D.; Thomas, H. J. J. Org. Chem. 1975, 40, 1923.

(b) Montgomery, J. A.; Thomas, H. J. J. Org. Chem. 1978, 43, 541.

[3] (a) Nair, V.; Jahnke, T. S. Antimicrob. Agents Chemother. 1995, 39, 1017.

(b) Nair, V.; Piotrowska, D. G.; Okello, M.; Vadakkan, J. Nucleosides Nucleotides Nucleic Acids 2007, 26, 687.

(c) Chun, B. K.; Vadakkan, J. J.; Nair, V. Nucleosides Nucleotides Nucleic Acids 2005, 24, 725.

[4] (a) Ogino, T.; Sato, K.; Matsuda, A. ChemBioChem 2010, 11, 2597. (b) Kira, T.; Kakefuda, A.; Shuto, S.; Matsuda, A.; Baba, M.; Shigeta, S. Nucleosides Nucleotides Nucleic Acids 1995, 14, 571.

(c)Yoshimura, Y.; Asami, K.; Matsui, H.; Tanaka, H.; Takahata, H. Org. Lett. 2006, 8, 6015.

[5] (a) Yu, H. W.; Zhang, H. Y.; Yang, Z. J.; Min, J. M.; Ma, L. T.; Zhang, L. H. Pure App. Chem. 1998, 70, 435.

(b) Tian, X. B.; Min, J. M.; Zhang, L. H. Tetrahedron: Asymmetry 2000, 11, 1877. 
(c) Yu, H. W.; Zhang, L. R.; Zhou, J. C.; Ma, L. T.; Zhang, L. H. Bioorg. Med. Chem. 1996, 4, 609.

[6] (a) Song, Y.; Yang, R.; Ding, H.; Sun, Q.; Xiao, Q.; Ju, Y. Synthesis-Stuttgart 2011, 1213.

(b) Sun, Z. D.; Zhu, Y. L.; Huang, H. Y.; Song, X. R.; Xiao, Q. Chin. J. Org. Chem. 2016, 36, 2729 (in Chinsese). (孙志东, 朱云龙, 黄海洋, 宋贤荣, 肖强, 有机化学, 2016, 36, 2729.)

[7] Huang, Y.; Chen, Z.; Chen, Y.; Zhang, H.; Zhang, Y.; Zhao, Y.; Yang, Z.; Zhang, L. Bioconjugate Chem. 2013, 24, 951.

[8] Cai, B.; Yang, X.; Sun, L.; Fan, X.; Li, L.; Jin, H.; Wu, Y.; Guan, Z.; Zhang, L.; Zhang, L.; Yang, Z. Org. Biomol. Chem. 2014, 12, 8866.

[9] Fan, X.; Sun, L.; Li, K.; Yang, X.; Cai, B.; Zhang, Y.; Zhu, Y.; Ma, Y.; Guan, Z.; Wu, Y.; Zhang, L.; Yang, Z. Mol. Ther-Nucleic Acids 2017, 9, 218.

[10] Li, L.; Yang, X.; Li, K.; Zhang, G.; Ma, Y.; Cai, B.; Li, S.; Ding, H.; Deng, J.; Nan, X.; Sun, J.; Wu, Y.; Shao, N.; Zhang, L.; Yang, Z. Org. Biomol. Chem. 2018, 16, 7488 .

[11] (a) Zhang, S.; Cao, M.; Guan, Z.; Wang, Z.; Cao, Y. L.; Guo, Y.; Yang, Z. J.; Zhang, L. H. Chin. J. Med. Chem. 2011, $2,4$.

(b) Zhang, S. M.S. Thesis, Peking University, Beijing, 2011 (in Chinese).

(张炼, 硕士论文, 北京大学, 北京, 2011.)

(c) Zhang, H. Y.; Wu, X. J.; Yu, H. W.; Ma, L. T.; Zhang, L. H. Chin. Chem. Lett. 1996, 7, 1089.

(d) Zhang, H. Y.; Zhang, M. L.; Piao, Z. S.; Ma, L. T.; Zhang, L. H. Acta Pharm. Sin. 1999, 34, 363 (in Chinese).

(张虎翼，张铭龙，朴志松，马灵台，张礼和，药学学报，1999, 34, 363.)

[12] (a) Zhang, P. S.; Dong, E Z. M.; Cleary, T. P. Org. Process Res. Dev. 2005, 9, 583. (b) Forsman, J. J.; Waerna, J.; Murzin, D. Y.; Leino, R. Eur. J. Org. Chem. 2009, 5666.

[13] CCDC 1892867 (Compound 8) contain the supplementary crystallographic data for this paper.

[14] Houston, T. A.; Koreeda, M. Carbohydr. Res. 2009, 344, 2240.

[15] Kakefuda, A.; Shuto, S.; Nagahata, T.; Seki, J.; Sasaki, T.; Matsuda, A. Tetrahedron 1994, 50, 10167.

[16] Ohrui, H.; Waga, T.; Meguro, H. Biosci. Biotechnol. Biochem. 1993, 57, 1040 .

[17] (a) Yoshimura, Y. Heterocycles 2017, 94, 1625.

(b) Kitkowska, J. D.; Tabaczynska, Z. A.; Draminski, M. Wiad. Chem. 2013, 67, 843.

(c) Leclerc, E. In Chemical Synthesis of Carbocyclic Analogues of Nucleosides, John Wiley \& Sons, Inc., New York, 2013, pp. 535 604.

[18] (a) Jacobsen, M. F.; Knudsen, M. M.; Gothelf, K. V. J. Org. Chem. 2006, 71, 9183 .

(b) Mercurio, M. E.; Tomassi, S.; Gaglione, M.; Russo, R.; Chambery, A.; Lama, S.; Stiuso, P.; Cosconati, S.; Novellino, E.; Di Maro, S.; Messere, A. J. Org. Chem. 2016, 81, 11612.

(c) Zhou, J.; Du, X.; Chen, X.; Xu, B. Biochemistry 2018, 57, 4867. (d) Porcheddu, A.; Giacomelli, G.; Piredda, I.; Carta, M.; Nieddu, G. Eur. J. Org. Chem. 2008, (34), 5786.

[19] (a) Tzeng, C.-C.; Hwang, L.-C.; Chen, C.-C.; Wei, D.-C. Nucleoside Nucleotides 1995, 14, 1425.

[20] Meade, E. A.; Wotring, L. L.; Drach, J. C.; Townsend, L. B. J. Med. Chem. 1997, 40, 794.

[21] Lenagh-Snow, G. M. J.; Araujo, N.; Jenkinson, S. F.; Rutherford, C.; Nakagawa, S.; Kato, A.; Yu, C.-Y.; Weymouth-Wilson, A. C.; Fleet, G. W. J. Org. Lett. 2011, 13, 5834. 